\title{
Sunitinib Induced Pyoderma Gangrenosum-Like Ulcerations
}

\author{
S. Akanay-Diesel ${ }^{1}$, N. P. Hoff ${ }^{1}$, S. Kürle ${ }^{1}$, J. Haes ${ }^{2}$, A. Erhardt ${ }^{2}$, D. Häussinger², K-W. Schulte ${ }^{1}$, \\ E. Bölke ${ }^{3}$, C. Matuschek ${ }^{3}$, W. Budach ${ }^{3}$, P. A. Gerber ${ }^{1}$, B. Homey ${ }^{1}$ \\ ${ }^{1}$ Department of Dermatology, ${ }^{2}$ Department of Gastroenterology, Hepatology and Infectiology, ${ }^{3}$ Department of Radiation Oncology, \\ Heinrich-Heine-Universität Duesseldorf, Germany
}

\begin{abstract}
Pyoderma gangrenosum is a non-infectious neutrophilic skin disease commonly associated with underlying systemic diseases. Histopathological and laboratory diagnostics are unspecific in the majority of the cases and the diagnosis is made in accordance with the clinical picture. Here, we report the case of a 69-year old man with progredient pyoderma gangrenosum-like ulcerations under treatment with sunitinib due to hepatocellular carcinoma. A conventional ulcer therapy did not lead to a regression of the lesions. Solely cessation of sunitinib therapy resulted in an improvement of the ulcerations. Sunitinib is a multikinase inhibitor that targets the PDGF- $\alpha-$ and $-\beta-$, VEGF-1-3-, KIT-, FLT3-, CSF-1- and RET-receptor, thereby impairing tumour proliferation, pathological angiogenesis and metastasation. Here, we demonstrate that pyoderma gangrenosum-like ulcers may represent a serious side effect of sunitinib-based anti-cancer treatment.
\end{abstract}

\section{INTRODUCTION}

Pyoderma gangrenosum (PG) is a non-infectious neutrophilic dermatosis and is often associated with an underlying systemic disease [5]. Diagnostic findings are unspecific in the majority of the cases and the diagnosis is made in accordance with the clinical picture. Often the inflammatory process is initiated by unspecific mechanical trauma.

Here, we report a case of PG like ulcerations under treatment with sunitinib.

\section{CASE REPORT}

A 69-year old man presented to our outpatient clinic with therapy refractory ulcerations of the right lower leg, which had been progredient over the last 4 months (Fig. 1). At the initial presentation we noticed multiple ulcerations (appr. $2 \times 2$ to $4 \times 6 \mathrm{~cm}^{2}$ ), hyperceratotical plaques as well as serous to purulent crusts. Moreover, the patient exposed clinical signs of chronic venous insufficiency, such as purpura jeaune d'ocre, corona phlebectatica paraplantaris and edema.

Comorbities included a metastasized hepatocellular carcinoma (HCC; inital diagnosis in 2007; pulmonary and adrenal metastases), an associated hepatitis $\mathrm{C}$, hepatic cirrhosis (CHILD A), hypothyreosis, arterial hypertonia and benign hyperplasia of the prostate gland.

The further patients medical history included a ra- diation of adrenal gland for metastases of the HCC two years ago, multiple transarterial chemoembolisations one year ago, palliative chemotherapies with gemcitabine and bevacizumab (for 5 months) two years ago and a therapy with sorafenib until four months before presentation in our clinic. At the initial presentation the patient had been receiving a palliative chemotherapy with sunitinib (37.5 mg/day p.o.).

Differential diagnoses for the ulcerations included arterial and venous ulcerations, PG, vasculitic ulcerations and traumatic or drug-related ulcerations. Yet, the characteristic signs of chronic venous insufficiency suggested chronical venous ulcerations as the most likely diagnosis.

Accordingly, we initiated a conventional ulcer therapy including topical antiseptic measures (octenidin solution), mometasonfuroat ointment for the area surrounding the ulcers, enzymatic debridement and foam dressings, as well as intermittant curretage of fibrinous coatings combined with compression therapy for a period of four months. However, these measures did not result in an improvement of the lesions. Conversely, the ulcerations showed a progress in size up to a maximum wound area of approximately $25 \times 30 \mathrm{~cm}^{2}$. Moreover, new ulcerations developed on the left lower leg (approx. $2 \times 2 \mathrm{~cm}^{2}$ ).

Diagnostic procedures included serological and hematological tests (hemoglobin $8.9 \mathrm{~g} / \mathrm{dl}$, leucocytes $2300 / \mu$ l, thrombocytes $152000 / \mu 1$, GOT $68 \mathrm{U} / 1, \mathrm{GPT}$ $49 \mathrm{U} / \mathrm{l}$, GGT $74 \mathrm{U} / 1$, CRP $0,9 \mathrm{mg} / \mathrm{dl}$, creatinine 0.8 $\mathrm{mg} / \mathrm{dl}$, AFP $1965 \mu \mathrm{g} / \mathrm{l}$, CA19-9 29.2 U/ml, pANCA and cANCA negativ), microbiological swabs, which revealed a generous amount of Klebsiella oxytoca, epicutaneous testing (including ointments and dressings, preservative agents, disinfectants, stabilizers, antioxidants and the european standard) and abdominal sonography, which showed hepatic cirrhosis, hepatocellular carcinoma lesions without progress at that time, splenomegalia, a metastasis of the adrenal gland and an enlarged prostate gland. Doppler ultrasound of both legs showed no significant insufficiency of veins. Because of serological (elevating CRP) and clinical signs of infection of the ulcerations we initiated a therapy with piperacillin plus combactam i.v., in accordance with the resistogramme. Concomitantly erythrocyte were given several times due to anemia (hemoglobin $<7 \mathrm{~g} / \mathrm{dl})$.

Next, we sought to perform vacuum assisted therapy to improve wound granulation and reduce bacterial 


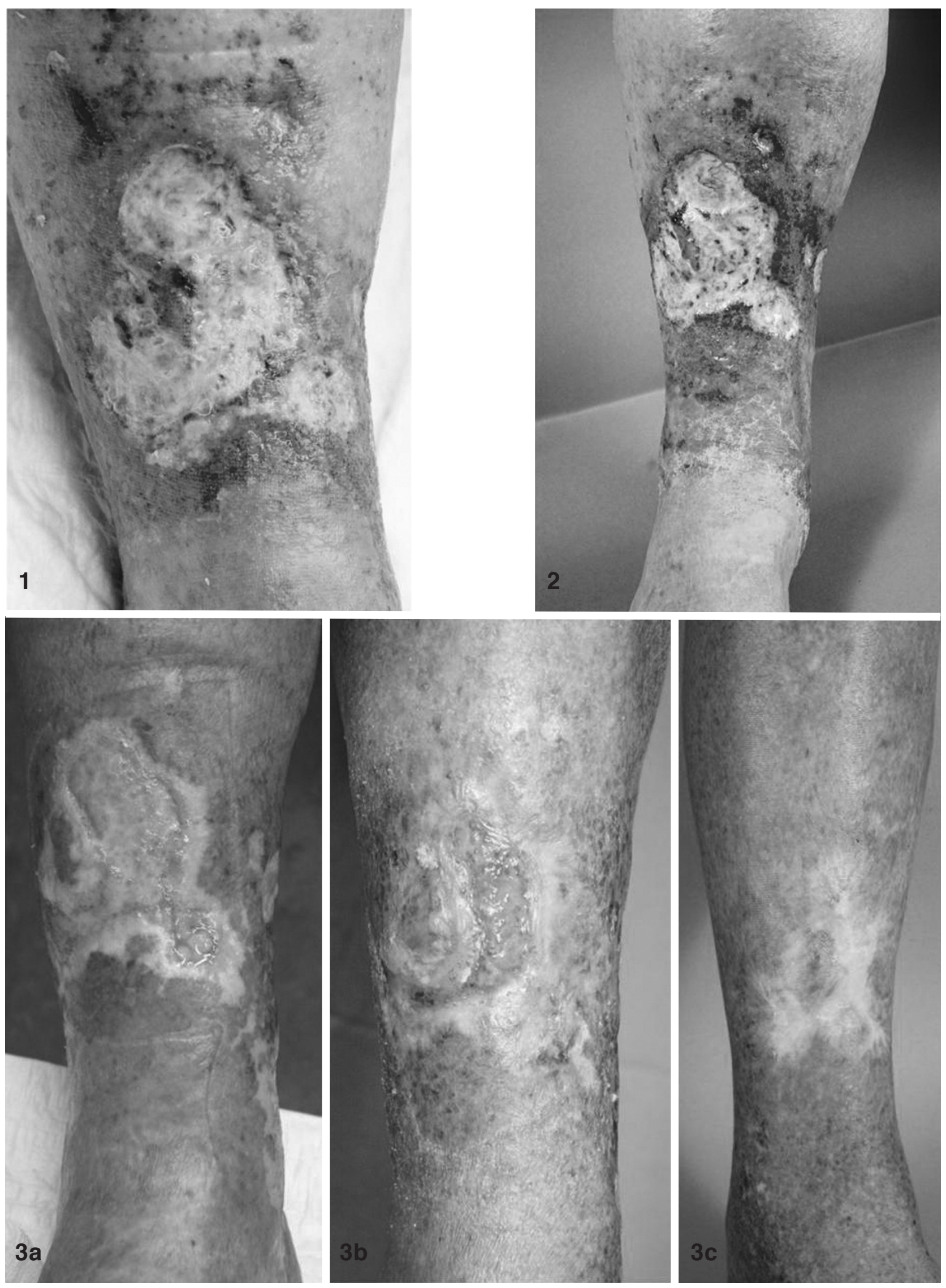

Fig. 1. Exsisting ulceration since four months of the right lower leg.

Fig. 2. Progress in size and new purpuric lesions after vacuum assisted therapy.

Fig. $3 a-c$. (a) One month, (b) two months, (c) six months after stopping treatment with sunitinib. 




Fig. 4. Hyperpigmentation of the skin.

colonization (white foam, $125 \mathrm{mmHg}$ ). Strikingly, after three days of vacuum therapy ulcerations showed a rapid progress in size and the development of additional purpuric lesions surrounding the ulcerations along with a livid undermining of the ulcer-borders was noticed (Fig. 2).

Subsequently performed histopathological examinations were unspecific and showed a compact corneal layer, unregularly broadened epithelia with hypergranulosis, dilated blood vessels in corium with on the one hand perivascular on the other hand interstitial infiltration consisting of lymphocytes and histiocytes and deposition of hemosiderin.

Based on the characteristic morphological signs, the histopathologic examination and the induction by minimal trauma the diagnosis of PG-like ulcerations was made. Moreover, the timely correlation of the beginning of ulcerations and the initiation of sunitinib therapy suggested a causal mechanism. Therefore, sunitinib was stopped in accordance with the gastroenterological departement. One month after stopping administration of sunitinib sorafenib therapy was initiated.

In addition, we performed a topical therapy, including desinfection with polyhexanide, $0.1 \%$ tacrolimus ointment, foam dressings and compression therapy [12].

Under the above mentioned treatment ulcerations progrediently improved. Six months after stopping treatment with sunitinib a nearly complete healing of the ulcerations was observed (Fig. 3a-c).

\section{Discussion}

PG is a non-infectious neutrophilic dermatosis that is characterized by pustulations that progress into painful ulcerations. Yet, the diagnosis is challenging and regarded a diagnosis of exclusion. Notably, PG is commonly initiated or aggravated by unspecific trauma. Accordingly, in our case vacuum assisted therapy and surgical debridement resulted in disease progression. PG is commonly associated with inflammatory bowel diseases, autoimmune diseases or malignancies

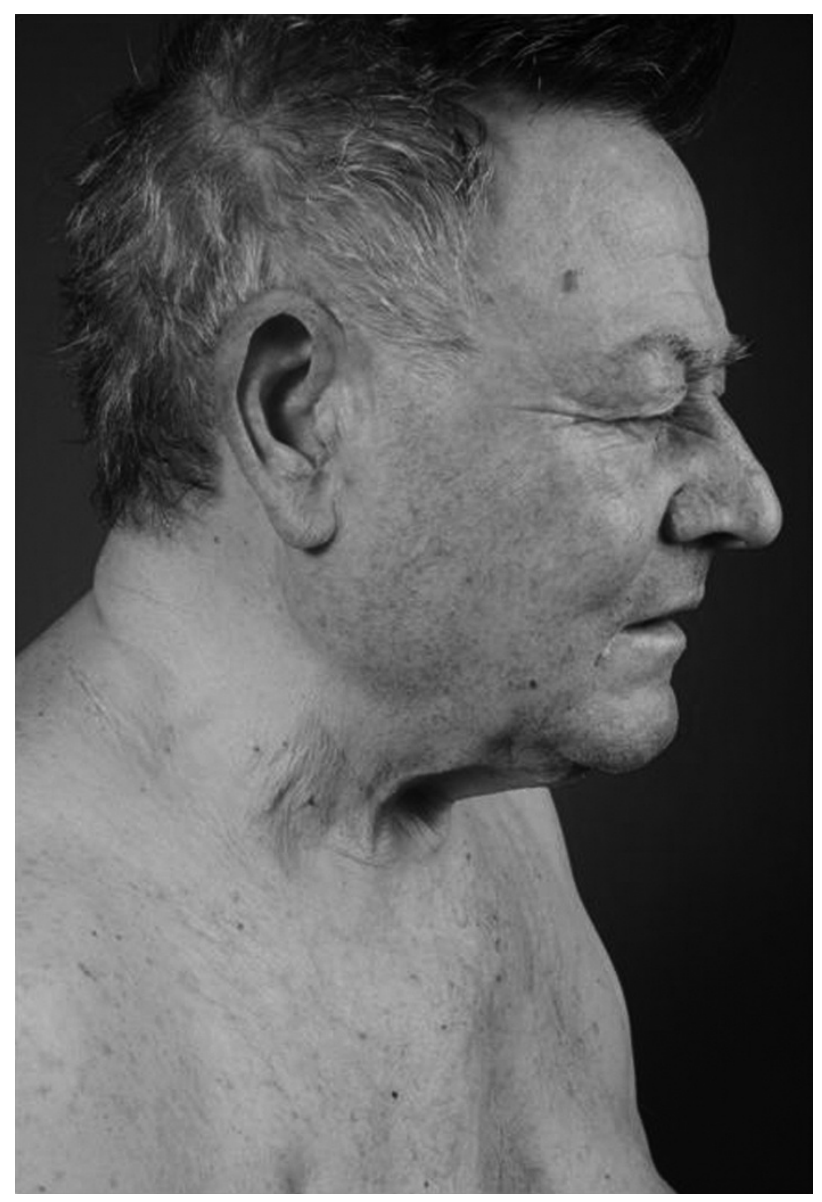

Fig. 5. Facial erythema and hypopigmentation of hair.

[13]. Therapy includes treatment of underlying systemic disease and if not contraindicated systemic immunosuppressive treatment with e.g. glucocorticosteroids or cyclosporine.

Sunitinib is a multikinase-inhibitor, which inhibits the PDGF- $\alpha$ - and $-\beta$-, VEGF-1-3-, KIT-, FLT3, CSF1 - and RET-receptor, thereby impairing tumour proliferation, pathological angiogenesis and metastazation. 
Sunitinib is used in the treatment of various tumor entities, such as metastasized renal cell carcinoma, gastrointestinal stroma tumour or HCC [14]. Known side effects of sunitinib include fatigue, diarrhoea, arterial hypertension, hypothyreosis and also cutaneous side effects like stomatitis, hypo- and hyperpigmentation, erythrodysesthesia palmoplantaris, facial edema, subungual hemorrhagia, facial erythema, alopecia and pyoderma gangrenosum-like ulcerations. In our patient we observed hyperpigmentation of the skin (Fig. 4), hypopigmentation of the hair as well as facial erythema (Fig. 5).

With regard to PG in our patient we could observe a rapid improvement of ulcerations after stopping treatment with sunitinib. In fact, we noticed an improvement of $80 \%$ in the first 3 months and a complete healing after 12 months (Fig 3c).

Today the cellular and molecular mechanism of PG-like ulcerations due to sunitinib have remained largely elusive but it has been suggested that the antiangiogenic effects of sunitinib by c-KIT- and VEGFR-inhibition induce necrosis and the subsequent invasion of neutrophilic granulocytes, hence initiating PG. Interestingly, also other tyrosine-kinase-inhibitors such as imatinib or erlotinib have been associated to neutrophilic dermatoses, such as sweet syndrome or papulopustular rash, suggesting common mechanisms $[9,10,11]$.

In summary, we here report a rare case of PG-like ulcerations due to sunitinib-based anti-cancer treatment and highlight probable management options. Further studies are needed to determine the exact mechanisms of sunitinib-associated adverse effects. [1, 2].

\section{REFERENCES}

1. Ten Freyhaus K, Homey B, Bieber T, Wilsmann-Theis D (2008) Pyoderma gangrenosum: another cutaneous side effect of sunitinib? Br J Dermatol. 2008 Jul;159(1):242-3

2. Dean SM, Zirwas M (2010) A second case of Sunitinibassociated Pyoderma Gangrenosum. J Clin Aesthet Dermatol. 2010 August; 3(8):34-35

3. Hartmann JT, Kanz L (2008) Sunitinib and periodic hair depigmentation due to temporary c-KIT inhibition. Arch Dermatol. 2008 Nov;144(11):1525-6.
4. Faivre S, Demetri G, Sargent W, Raymond E (2007) Molecular basis for sunitinib efficacy and future clinical development. Nat Rev Drug Discov. 2007 Sep;6(9):734-45

5. Hoff NP, Bölke E, Matuschek C, Peiper M, Neumann NJ, Gerber PA (2010) Pyoderma gangraenosum. Wien Klin Wochenschr. 2010 Feb;122(3-4):75

6. Nadauld LD, Miller MB, Srinivas S (2010) Pyoderma Gangrenosum With the Use of Sunitinib. J Clin Oncol 2010, Vol 28

7. Nguyen S, Franklin M, Dudek AZ Skin ulcers in KlippelTrenaunay syndrome respond to sunitinib. (2008) Transl Res. 2008 Apr

8. Nadauld LD, Miller MB, Sriniva S (2011) Pyoderma Gangrenosum With the Use of SunitinibJournal of Clinical Oncology, 2011, Vol 29

9. Neoh CY et al Sweet's syndrome: a spectrum of unusual clinical presentations and associations. Br J Dermatol. 2007 Mar;156(3):480-5.

10. Gerber PA et al Therapie mit Inhibitoren des epidermalen Wachstumsfaktorrezeptors. Hautarzt 2010

11. Lübbe et al. Spongiosis induced by Erlotinib (Tarceva $\left.{ }^{\circledR}\right)$. Dermatology 2008, 216:247-249

12. Marquardt $C$ et al. Evaluation of the tissue toxicity of antiseptics by the hen's egg test on the chorioallantoic membrane (HETCAM). Eur J Med Res. 2010 May 18;15(5): 204-9.

13. Harati A et al. Skin disorders in association with monoclonal gammopathies. Eur J Med Res. 2005 Mar 29;10(3): 93-104.

14. Staehler $\mathrm{M}$ et al. Carcinoma of the collecting ducts of Bellini of the kidney: adjuvant chemotherapy followed by multikinase-inhibition with sunitinib. Eur J Med Res. 2008 Nov 24;13(11):531-5.

Received: June 6, 2011 / Accepted: July 7, 2011

Address for correspondence:

PD. Dr. med. Edwin Bölke

Universitätsklinikum Düsseldorf

Heinrich Heine Universität

Moorenstrasse 5

40225 Duesseldorf

Germany

Tel.: $\quad+49-221-81-17990$

Fax: +49-211-81-17051

E-mail: boelke@med.uni-duesseldorf.de 